\title{
An unsupervised framework for tracing textual sources of moral change
}

\author{
Aida Ramezani ${ }^{1 *}$, Zining Zhu ${ }^{1,2 *}$, Frank Rudzicz ${ }^{1,2,3}$, Yang $\mathrm{Xu}^{1,2,4}$ \\ ${ }^{1}$ Department of Computer Science, University of Toronto, Toronto, Canada \\ ${ }^{2}$ Vector Institute for Artificial Intelligence, Toronto, Canada \\ ${ }^{3}$ St. Michael's Hospital, Toronto, Canada \\ ${ }^{4}$ Cognitive Science Program, University of Toronto, Toronto, Canada \\ \{armzn, zining, frank, yangxu\}@cs.toronto.edu
}

\begin{abstract}
Morality plays an important role in social wellbeing, but people's moral perception is not stable and changes over time. Recent advances in natural language processing have shown that text is an effective medium for informing moral change, but no attempt has been made to quantify the origins of these changes. We present a novel unsupervised framework for tracing textual sources of moral change toward entities through time. We characterize moral change with probabilistic topical distributions and infer the source text that exerts prominent influence on the moral time course. We evaluate our framework on a diverse set of data ranging from social media to news articles. We show that our framework not only captures fine-grained human moral judgments, but also identifies coherent source topics of moral change triggered by historical events. We apply our methodology to analyze the news in the COVID-19 pandemic and demonstrate its utility in identifying sources of moral change in high-impact and real-time social events.
\end{abstract}

\section{Introduction}

From ancient Greek scholars to philosophers of the past centuries, morality has been a subject of central importance in human history (Plato and Bloom, 1968; Aristotle et al., 2009; Hume, 1739; Smith, 1759; Kant, 1785; Nietzsche, 1887). Despite this importance, people's morals are not static but change over time (Bloom, 2010). Recent advances in natural language processing (NLP) have shown that text can inform moral sentiment and its change over time (e.g., how slavery was increasingly perceived to be morally wrong) (Xie et al., 2019; Garten et al., 2016). However, critically under-explored are the origins of these changes. We present a framework for tracing textual sources of moral change that requires minimal human intervention or supervision.
The study of moral sentiment is a prominent subject in social psychology (Piaget, 1932; Kohlberg, 1969; Kohlberg and Hersh, 1977; Haidt, 2001; Pizarro and Bloom, 2003), and the advent of Moral Foundations Theory (Graham et al., 2013) has provided an impetus for text-based analysis of moral sentiment in natural language processing. Existing studies range from moral sentiment classification to temporal inference of moral sentiment change (e.g., Garten et al., 2016; Mooijman et al., 2018; Lin et al., 2018; Xie et al., 2020, 2019).

The problem we focus on here is how moral perception toward entities (e.g., political leaders) varies through time, and whether textual analysis can help extract the sources of this variation. For instance, an entity like Bill Clinton could be applauded for charity at one time but deprecated for a sex scandal at another time. Similarly, moral sentiment toward a more general entity like policemen could undergo a negative shift due to acts on racial discrimination. Existing methods for moral sentiment detection typically take an aggregate approach and do not focus on analyzing moral sentiment of entities (Garten et al., 2016; Lin et al., 2018; Mooijman et al., 2018; Xie et al., 2019). Here, we develop a methodology to identify textual sources that give rise to moral sentiment change toward an entity. Our work takes a similar approach to detecting sources of gender bias in text by locating a set of documents that influence gender bias in word embeddings (Brunet et al., 2019).

We propose a probabilistic unsupervised framework informed by both textual inference of moral sentiment and dynamic topic model (Blei and Lafferty, 2006). Capturing events as topic distributions, we approach this problem by decomposing textual mentions of an entity into topics and quantifying the contributions of different topics toward moral sentiment of an entity. We attribute the origins of moral change as topics that contribute saliently to changes in the time course of moral 
a)

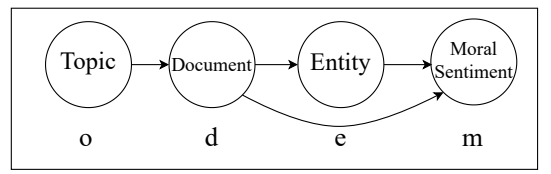

b)

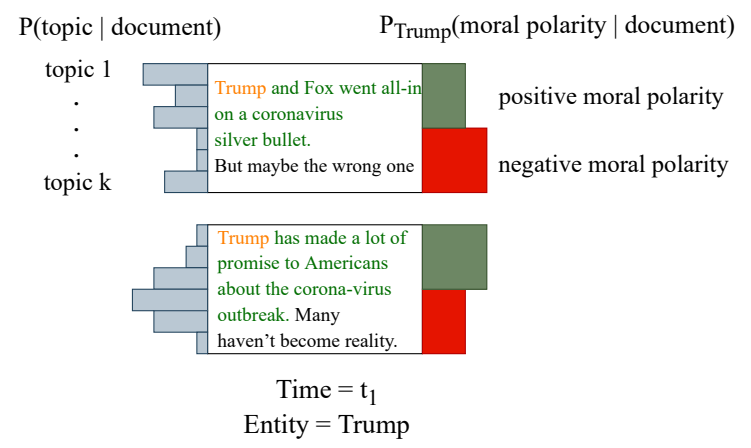

Figure 1: Graphical model and illustration of our framework for topic-based source analysis of moral change.

sentiment. We compare this approach with classic work on influence function (Cook and Weisberg, 1980), which has been used to quantify the effect of samples in statistical estimation (see also Koh and Liang, 2017; Brunet et al., 2019).

Figure 1 illustrates our framework. Figure 1a shows the generative process in our topic-based approach. Given an entity (e.g., Donald Trump) and its mentions in a set of documents (e.g., tweets or news articles), we wish to infer the most salient source topic(s) that gave rise to changes in the moral sentiment time course about that entity. Here as an illustration the moral sentiment toward Donald Trump is analyzed through a set of news articles. Each article includes mentions of this entity, specified as topical distributions that contribute toward the perceived moral sentiment of the entity (see Figure 1b). As moral sentiment of the entity changes over time, our framework uses probabilistic inference jointly with change point analysis to extract the most salient topic and its relevant source documents that underlie these changes. We show how our approach predicts fine-grained human moral judgment variation across topics and identifies influential and coherent text as the sources of moral change for both historical and modern events.

\section{Related work on textual inference of moral sentiment in NLP}

The development of Moral Foundations Theory (MFT) jointly with Moral Foundations Dictionary (MFD) (Graham et al., 2009, 2013) has propelled recent research in the natural language processing community to explore automated textual inference of moral sentiment. MFT sought to explain the cultural variation in morality and moral concerns along five or six moral foundations, each organized in terms of the polarities virtue (+) and vice (-).

The computational methods using MFT tend to rely on supervised approaches to predicting the moral sentiment reflected in text (Garten et al., 2016; Lin et al., 2018; Mooijman et al., 2018; Xie et al., 2020). Other related work has characterized moral biases in language models (Schramowski et al., 2019; Jentzsch et al., 2019; Xie et al., 2019), and contributed new datasets for tasks such as automatic ethical judgment and inference of sociomoral norms (Hoover et al., 2020; Lourie et al., 2020; Forbes et al., 2020). Existing work has also studied moral sentiment change over time (Xie et al., 2019) showing how word embeddings capture hidden moral biases underlying different concepts (e.g., slavery) in history. This model uses MFD words as seeds and a hierarchical framework to capture moral change in three tiers: moral relevance, moral polarity, and fine-grained moral foundations. ${ }^{1}$

Here we go beyond this line of research by developing an unsupervised framework that automatically identifies sources of moral change toward entities in text.

\section{Methodology}

We formulate textual source tracing of moral change as a probabilistic inference problem. This model allows us to identify the sources of change at the topic level (source topic), as well as retrieve a set of related documents (source documents) underlying the detected moral change. To do so, we need to quantify 1) the moral time course of an entity based on textual input, and 2) the influence of topics on the changes in moral time course. ${ }^{2}$

Quantification of moral time course. We estimate the moral sentiment along moral dimension $m$ for entity $e$ at time point $t$ as follows:

$$
P(m \mid e, t)=\frac{\sum_{d \in D_{e, t}} P_{e}(m \mid d)}{\left|D_{e, t}\right|}
$$

\footnotetext{
${ }^{1}$ The 10 foundation categories follow from the Moral Foundations Theory including 5 opposing pairs: $\quad \mathrm{Care}(+) / \operatorname{Harm}(-), \quad$ Fairness $(+) /$ Cheating $(-)$, Loyalty (+)/Betrayal $(-), \quad$ Authority $(+) / \operatorname{Subversion}(-)$, and Sanctity(+)/Degradation(-).

${ }^{2}$ Codes to replicate the analyses are publicly available at https://github.com/AidaRamezani/ moral-source-tracing
} 
Here $D_{e, t}$ is the set of documents (indexed by $d$ ) at time point $t$ that contain entity $e$ at least once. ${ }^{3}$ For example, $D_{e, t}$ can be all the documents in our corpus that are published in $t=$ December 1997, and include a mention of entity $e=$ Bill Clinton. Moral dimension $m$ can be moral relevance, moral polarity, or one of the moral foundations in MFT.

To construct a vector representation for a document, we exclude all the sentences in $d$ that do not include any mentions of entity $e$. After lemmatizing the rest of the document using spaCy English model, we remove 1) function words, 2) entity $e$ and its mentions, and 3) words that are classified as morally irrelevant by the centroid model following Xie et al. (2019). We then derive the vector representation of document $d$ by taking an average of the semantic vector representations (i.e., word embeddings) of the remaining words: $V_{d}=\frac{1}{|d|} \sum_{w \in d} V_{w}$. Here $V_{d}$ is the vector representation of document $d$, and $V_{w}$ is the vector representation of lemma $w$.

To estimate $P_{e}(m \mid d)$, we use the centroid model in Xie et al. (2019). This model estimates this probability by comparing the similarity of an input vector (i.e., $V_{d}$ ) to each of its centroids. The centroids of this model in the moral relevance tier are the average word embeddings of MFD words and a set of morally neutral words. For moral polarity, the centroids are based on moral virtue and vice words from MFD. For the fine-grained tier, there are 10 centroids, each being the average word embeddings of the words in a moral foundation.

Quantification of textual source and influence of moral change. To quantify sources of moral change, we first use the dynamic topic model (Blei and Lafferty, 2006) to infer emerging topics based on the temporal collection of documents that contain entity $e$ (illustrated in Figure 1). Using a dynamic topic model offers the flexibility to update old and dated topics with emerging topics over time. For entity $e$ with $k$ associated topics, we then define metric $\Delta S$ to quantify the influence of each topic on moral change toward this entity in time window $t \sim t+\Delta t$ (excluding $t$ ). Similar to Equation $1, t$ is a point in time, e.g., December 1997, and $\Delta t$ is a time period, e.g., 3 months. Formally,

\footnotetext{
${ }^{3} \mathrm{We}$ use the co-reference resolution module neuralcoref implemented in spaCy to find all the mentions of an entity in a document. We describe the details of the pre-processing in Appendix A.
}

this metric is as follows:

$$
\begin{gathered}
\Delta S(e, m, o, t, \Delta t)= \\
\mid P(m \mid e, t \sim t+\Delta t, \text { topic } \neq o)-P(m \mid e, t) \mid
\end{gathered}
$$

Here $o$ represents a topic ranging from 1 to $k$, and $\Delta S$ measures the degree to which removing a topic can restore the moral sentiment to its base state. The topic with the lowest $\Delta S$ is the most influential source for the change. We derive $P(m \mid e, t \sim t+$ $\Delta t$, topic $\neq o$ ) as follows:

$$
\begin{aligned}
P(m \mid e, t & \sim t+\Delta t, \text { topic } \neq o) \\
= & \sum_{d \in D_{e, t \sim t+\Delta t}} P_{e}(m \mid d) P(d \mid \text { topic } \neq o) \\
\propto & \sum_{d \in D_{e, t \sim t+\Delta t}} P_{e}(m \mid d) P(\text { topic } \neq o \mid d) \\
& \propto \sum_{d \in D_{e, t \sim t+\Delta t}} P_{e}(m \mid d)(1-P(\text { topic }=o \mid d))
\end{aligned}
$$

We estimate $P($ topic $=o \mid d)$ from the dynamic topic model. Similar to Equation $1, D_{e, t \sim t+\Delta t}$ represents the documents that contain entity $e$ appearing within time window $t \sim t+\Delta t$. Without loss of generality, we assume a uniform prior for the distribution of the documents, so $P(d)$ is constant.

We detect significant changes in moral time course using an established method for changepoint detection (Kulkarni et al., 2015). Given a time series as the input, this method first generates random perturbations of the time series and compares the magnitude of the mean shift before and after a time point in the original series to that in the random perturbations, for all the points in the time course individually. The outputs of the algorithm will be the time points with the most significant mean shifts (i.e., lowest p-values) as the change points. We consider a sliding window with a size of $W_{t}$ time points and a step size of $W_{s}$ and run the change point detection algorithm on the moral sentiment time series of an entity by estimating the probability in Equation 1 incrementally over time. This gives change point(s) $t$ and the relevant time window(s) $\Delta t$ which we use in Equations 2 and 4. We find $W_{t}=7$ and $W_{s}=3$ to be reasonable choices.

We define $\Delta J$ to quantify the degree of influence of a set of documents $D^{*}$ (appearing at time $t \sim t+\Delta t$ ) on moral change toward entity $e$ in moral dimension $m$ at time $t$. We compute this by 
calculating how the entity-based moral change is impacted by the removal of $D^{*}$, formally as:

$$
\begin{gathered}
\Delta J\left(e, m, D^{*}, t, \Delta t\right)= \\
\left|P\left(m \mid e, t \sim t+\Delta t, D_{e, t \sim t+\Delta t} \backslash D^{*}\right)-P(m \mid e, t)\right|
\end{gathered}
$$

Here $P\left(m \mid e, t \sim t+\Delta t, D_{e, t \sim t+\Delta t} \backslash D^{*}\right)$ is calculated using Equation 1 over the documents including entity $e$ appearing at $t \sim t+\Delta t$ excluding set $D^{*}$. The difference between $\Delta S$ and $\Delta J$ is that $\Delta S$ measures the influence of a topic over all the documents in a probabilistic setting, whereas $\Delta J$ measures the influence of a set of documents regardless of their topic associations.

\section{Experiments and results}

We evaluate and apply our framework in three diverse and real-world settings.

\subsection{Datasets}

Moral Foundations Twitter Corpus (MFTC). We use Moral Foundations Twitter Corpus (Hoover et al., 2020) for the first case study. This corpus provides a large set of human judgments along different moral dimensions for tweets divided into distinct topics. Each tweet is hand-annotated for the 10 foundation categories and moral relevance. Using Twitter Developer Account, we were able to extract 21,482 tweets falling under six topic domains specified in the original dataset: ALM (all lives matter), BLM (black lives matter), Baltimore, Davidson, Election, and Sandy.

New York Times Annotated Corpus (NYT). We use the New York Times Annotated Corpus (Sandhaus, 2008) for the second case study. This dataset contains over 1.8 million news articles published in the New York Times from 1987 to 2007.

COVID-19 News Dataset (COVID). We use AYLIEN Free Coronavirus Dataset in the third case study. ${ }^{4}$ This dataset contains more than 1,500,000 annotated English news articles relevant to the COVID-19 pandemic. We include the articles published in well-known United States news agencies from January, 2020 to the end of July 2020. We extracted a total number of 94,732 articles from CNN, Foxnews, NBC News, The New York Times, USA Today, abc News, CBS News, Washington Post, MSNBC News, and Los Angeles Times.

\footnotetext{
${ }^{4}$ https: / / aylien.com/blog/ free-coronavirus-news-dataset
}

\subsection{Evaluation on human moral judgment}

Human moral sentiment toward entities may vary across topical contexts. As an initial study, we show how this variation is present in social media and can be captured by a topic-based approach where topic information is given. We use the MFTC tweet data for evaluation, based on the moral judgment of tweets in 6 topics: ALM, BLM, Baltimore, Davidson, Election, and Sandy. We summarize the human moral judgment of entities across topics using a count-based measure. Specifically, we compute the empirical probability $\widehat{P}(m \mid e, o)$ for moral dimension $m$, entity $e$, and topic $o$ as $\widehat{P}(m \mid e, o)=\frac{\operatorname{count}(m, e, o)}{\operatorname{count}(e, o)}$. Here $\operatorname{count}(e, o)$ is the number of tweets in topic $o$ that contain entity $e$. To calculate $\operatorname{count}(m, e, o)$, we count the number of tweets from topic $o$ that contain entity $e$, and were annotated with moral sentiment dimension $m$ in MFTC. To prepare ground-truth data, we take the following steps: 1) For the moral relevance dimension, if more than half of the annotators annotate a tweet "non-moral", we consider the tweet as morally irrelevant. 2) For the moral polarity dimension, if the majority of annotations fall under the positive fine-grained categories, the moral polarity of the tweet is positive (and negative otherwise). 3) For the foundation categories, each tweet is given the label of the category receiving the majority vote from the annotators. If more than one category satisfies this condition, we randomly assign one of them to the tweet. We also used graded proportions instead of binary ground-truth labels and obtained similar results. We analyzed moral judgment on the 53 most frequent entities in the MFTC that appear under at least two topics. The entities include hashtags, mentions, and the named entities such as people, organizations, groups, and concepts. $^{5}$

We first consider a topic-based model that explicitly uses topic information and applies static word embeddings to infer moral sentiment variation across topics. For each moral dimension $m$, entity $e$, and topic $o$ we derive the following probability using the methodology from Section 3: $P(m \mid e, o) \propto \sum_{d \in \text { tweets }} P_{e}(m \mid d) P(o \mid d)$. We use Word2Vec word embeddings (Mikolov et al., 2013) to represent each tweet as a single vector. We also

\footnotetext{
${ }^{5}$ The named entities are detected using the NER in spaCy. We manually check these entities, and map all the forms of an entity to a single unique type (e.g., Barack Obama and Obama are both considered the same entity).
} 


\begin{tabular}{lccc|cccc|ccc} 
& \multicolumn{3}{c}{$\begin{array}{c}\text { Topic-based Model } \\
\text { Moral Foundation }\end{array}$} & \multicolumn{3}{c}{$\begin{array}{c}\text { Topic-free Model } \\
\text { (Static Embedding) }\end{array}$} & \multicolumn{3}{c}{$\begin{array}{c}\text { Topic-free Model } \\
\text { (Static Embedding) }\end{array}$} & \multicolumn{3}{c}{ (Contextual Embedding) } \\
\cline { 2 - 11 } & $F_{1}$ & Pearson's r & $\mathrm{n}$ & $F_{1}$ & Pearson's r & $\mathrm{n}$ & $F_{1}$ & Pearson's r & $\mathrm{n}$ \\
\hline Moral Relevance & $\mathbf{1}$ & $\mathbf{0 . 3 0 7}$ & 195 & $\mathbf{1}$ & $0.098^{-}$ & 195 & $\mathbf{1}$ & $0.103^{-}$ & 195 \\
\hline Moral Polarity & $\mathbf{0 . 9 4 7}$ & $\mathbf{0 . 8 0 8}$ & 171 & $\mathbf{0 . 9 4 7}$ & 0.638 & 171 & 0.841 & 0.763 & 127 \\
\hline Authority & $\mathbf{0 . 9 2 4}$ & 0.285 & 157 & 0.689 & $0.199^{-}$ & 157 & 0.699 & $\mathbf{0 . 3 0 5}$ & 94 \\
Subversion & $\mathbf{0 . 8 7 7}$ & $\mathbf{0 . 2 5 1}$ & 143 & 0.705 & $-0.028^{-}$ & 143 & 0.777 & $0.242^{-}$ & 110 \\
Care & $\mathbf{0 . 9 2 4}$ & $\mathbf{0 . 5 0 0}$ & 157 & 0.689 & 0.328 & 157 & 0.699 & 0.451 & 94 \\
Harm & $\mathbf{0 . 8 7 7}$ & $0.060^{-}$ & 143 & 0.705 & $0.036^{-}$ & 143 & 0.777 & $\mathbf{0 . 2 8 6}$ & 110 \\
Fairness & $\mathbf{0 . 9 2 4}$ & $\mathbf{0 . 5 8 7}$ & 157 & 0.689 & 0.391 & 157 & 0.699 & 0.551 & 94 \\
Cheating & $\mathbf{0 . 8 7 7}$ & $\mathbf{0 . 3 4 1}$ & 143 & 0.705 & $0.193^{-}$ & 143 & 0.777 & $0.125^{-}$ & 110 \\
Loyalty & $\mathbf{0 . 9 2 4}$ & $\mathbf{0 . 6 3 4}$ & 157 & 0.689 & 0.524 & 157 & 0.699 & $0.236^{-}$ & 94 \\
Betrayal & $\mathbf{0 . 8 7 7}$ & $0.125^{-}$ & 143 & 0.705 & $0.045^{-}$ & 143 & 0.777 & $-0.104^{-}$ & 110 \\
Sanctity & $\mathbf{0 . 9 2 4}$ & $\mathbf{0 . 5 2 6}$ & 157 & 0.689 & 0.354 & 157 & 0.699 & 0.366 & 94 \\
Degradation & $\mathbf{0 . 8 7 7}$ & 0.386 & 143 & 0.705 & 0.434 & 143 & 0.777 & $\mathbf{0 . 5 2 4}$ & 110
\end{tabular}

Table 1: Evaluation of topic-based and topic-free models in predicting fine-grained human moral judgments, based on both $\mathrm{F}_{1}$ score and Pearson's correlation. Superscript minus sign under "Pearson's r" indicates $p>0.05$ (Bonferroni corrected).

consider two alternative topic-free models using static and contextual embeddings, where topic information is discarded in moral sentiment inference, i.e., $P(m \mid e, o)=P(m \mid e)$. We use BERT (Devlin et al., 2019) in the contextual embedding model to represent tweets. Similarly for the centroid model, instead of using the static embeddings of the seed words in MFD, we use BERT to embed their definitions from the online version of the Oxford English Dictionary (OED). ${ }^{6}$

Each model infers $P(m \mid e, o)$ for all entities, topics, and moral dimensions. We compare these probabilities with ground-truth moral judgments $\widehat{P}(m \mid e, o)$ using both $\mathrm{F}_{1}$ score and Pearson's correlation. We consider estimates of $\widehat{P}(m \mid e, o)$ and $P(m \mid e, o)$ meaningful if 1$)$ the entity appears in at least one of the tweets in topic $o$, and 2) there is at least one tweet in topic $o$ containing entity $e$ that satisfies the 3-tier hierarchical structure, i.e., moral polarity of an entity is only estimated when it is morally relevant, and virtuous/vice moral foundations sentiments are estimated only for morally positive/negative input. A correlation test is performed on the samples that satisfy the two criteria in both model and human judgment. $F_{1}$ score quantifies the proportion of samples that they agree on.

Table 1 summarizes our results in this task. We observe that the topic-based model best accounts for the variation in human moral judgment across topics for the entities analyzed, both in terms of the $F_{1}$ scores and fine-grained correlation values.

\footnotetext{
${ }^{6}$ https: // www. oed.com
}

For example, the entity USA bears an overall negative moral polarity, while the same entity appears more morally positive in tweets concerning the topic Election. Another example is that the entity $C N N$ displays a negative moral polarity across all topics, but shifts to a morally positive sentiment under the topic $A L M$. Our topic-based model with static embedding captures both of these variations. These initial results provide strong support to our presumption that moral sentiment toward entities may vary across context. We next apply our framework to diachronic data where neither topic information nor change point is provided.

\subsection{Evaluation on moral source identification from news of historical events}

In the second case study, we use the NYT dataset to evaluate the topic-based source model against prominent historical events in the United States from the $20^{\text {th }}$ and $21^{\text {st }}$ centuries, and analyze the entities associated with each event. We assess the topic-based source model on its ability to identify the moral changes at the historical incidents and locate topics and source text (i.e., news articles) relevant to these events. We also use the established influence function (Cook and Weisberg, 1980) as a baseline model for comparison.

Evaluation metrics. We consider a baseline inspired by influence function (Cook and Weisberg, 1980) to retrieve a set of documents as the textual source of moral change. We compare this set to the documents retrieved by the topic-based source 
a)

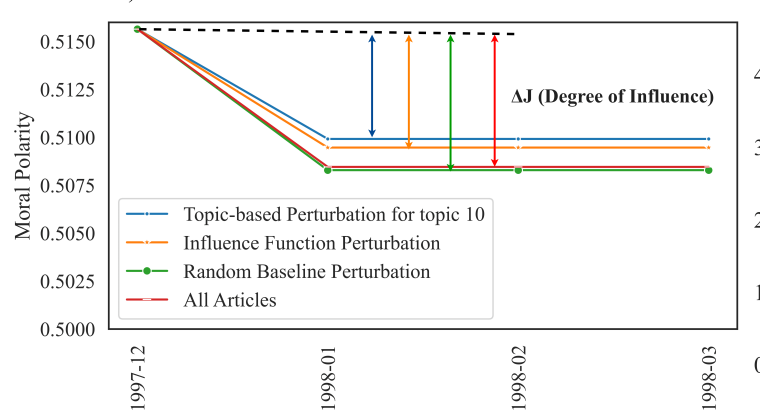

b)

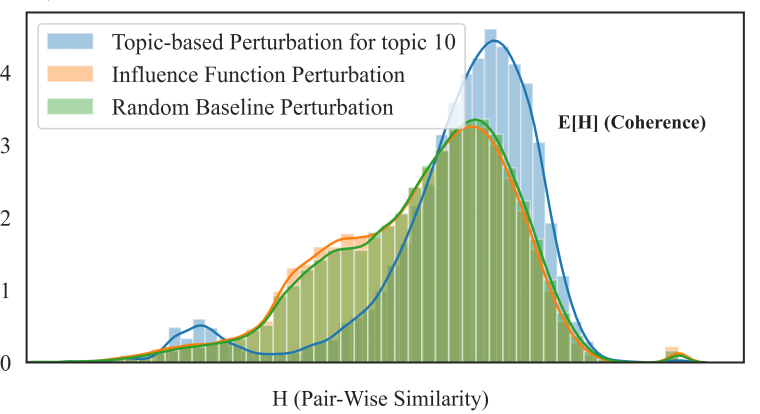

Figure 2: Illustration of metrics quantifying the effectiveness of models for tracing sources of moral sentiment change based on NYT news about Bill Clinton from 1997-12 to 1998-03. a) Comparing the degree of influence $(\Delta J)$ of different methods on restoring the change to its baseline (horizontal dash): smaller $\Delta J$ indicates greater influence. b) Expected coherence of source documents $(E[H])$ of different methods.

model based on the metrics of 1) degree of influence and 2) coherence of the retrieved source.

To assess models based on degree of influence, we use $\Delta J$. We first generate a null distribution via perturbing the dataset. The dataset used here is a set of documents published in $t \sim t+\Delta t$ that mention entity $e$ (i.e., $D_{e, t \sim t+\Delta t}$ ). To construct the null distribution, we choose a random set of documents from $D_{e, t \sim t+\Delta t}$, denoted as $D^{*}$, and measure the influence of set $D^{*}$ on moral sentiment at a change point using $\Delta J$ in Equation 4. We repeat this process until we generate 10,000 random document sets. The set of documents that minimizes $\Delta J$ significantly $(\alpha=0.05)$ compared to the null distribution would be the source text. These documents form a subset that provides the maximal perturbation to the moral sentiment estimated at the change point. For the topic-based model, we select the source set by choosing documents with the highest $p($ topic $=o \mid d)$, where $o$ is the topic minimizing Equation 2. The size of the source documents set for both the influence function and topic-based model would be $10 \%$ of $\left|D_{e, t \sim t+\Delta t}\right|$. We then compare $\Delta J$ of these two sets. A lower value for $\Delta J$ indicates greater influence and hence a more effective identification of the source documents.

We define $E[H]$ to assess the coherence in the retrieved source documents. $E[H]$ is the average pairwise cosine similarity among a set of retrieved documents (i.e., news articles in this case). We consider coherence a desirable property because the sources responsible for moral change toward an entity should ideally reflect a consistent set of content. The coherence metric evaluates whether the retrieved source documents indeed form a con- sistent set of text. We use Word2Vec embeddings to estimate the cosine similarities of news articles based on their headlines. Equation 5 defines this metric for a document set $D$. In this equation, $V_{h_{d_{i}}}$ corresponds to the vector representation of the headline of news article $d_{i}$. For both the topicbased model and influence function, $E[H]$ is estimated on the same set of documents as for $\Delta J$ :

$$
E[H]=\frac{1}{|D|(|D|-1)} \sum_{d_{i} \in D} \sum_{\substack{d_{j} \in D \\ i \neq j}} \frac{V_{h_{d_{i}}} \cdot V_{h_{d_{j}}}}{\left\|V_{h_{d_{i}}}\right\|\left\|V_{h_{d_{j}}}\right\|}
$$

We also consider a random baseline which arbitrarily retrieves the same number of documents as the topic-based and influence function methods.

Figure 2 illustrates $\Delta J$ and $E[H]$ based on NYT news about entity Bill Clinton from 1997-12 to 1998-03. The degree of influence and the coherence under the topic-based model are greater than those of the influence function and the random baseline. In particular, we observe that the topic retrieved as the source of the negative change in moral polarity of Bill Clinton is associated with the Clinton-Lewinsky Scandal (salient topic words include lawyer, Starr, Lewinsky, Jones), while the articles selected by influence function (salient words include plan, political, senate, patience) and the random baseline (sample words include Iraq, Democrat, world, battle) show minimal agreement in the context and no relevance to the ground-truth historical scandal of the period. The table in Appendix B shows the headlines of randomly sampled articles retrieved as sources of moral sentiment change by the three models.

For a more comprehensive evaluation, we se- 


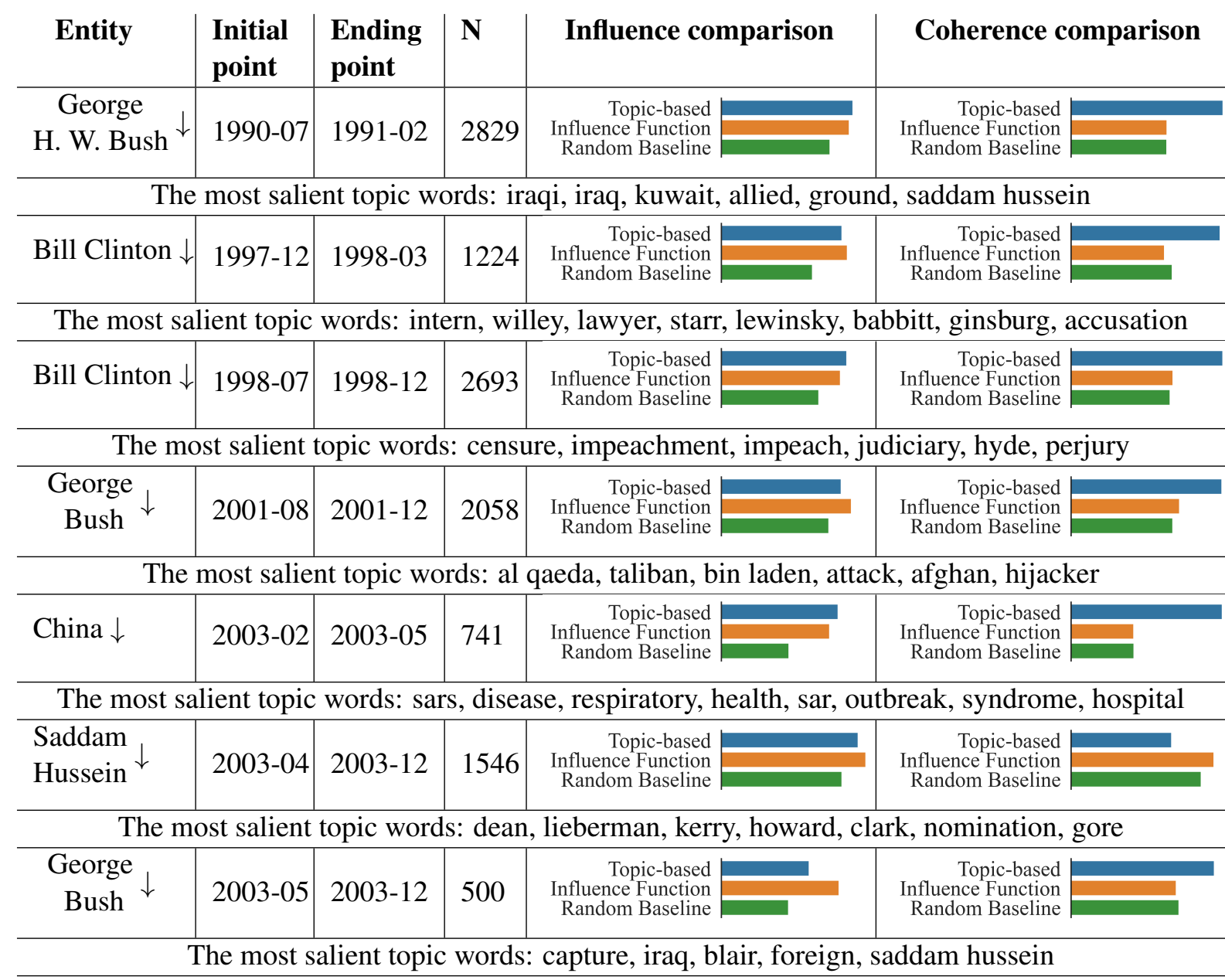

Table 2: Textual source analyses for moral change toward entities in historical events. Arrows show the directions of the moral polarity change. Column "N" shows the number of articles retrieved in each time window. The influence set size is $10 \%$ of $N$. Bars under "Influence comparison" show inverse $\Delta J_{\mathrm{s}}$ (lengthier for greater influence) under the three methods. "Coherence comparison" compares mean coherence $(E[H])$ of source text retrieved. The most salient words under the topic-based method are provided.

lect the following well-known historical events and entities: George H. W. Bush for Gulf War (19901991), Bill Clinton for the Clinton-Lewinsky Scandal (1997-1998), George W. Bush for September 11 attacks (2001), China for the SARS outbreak (20022004), George W. Bush and Saddam Hussein for the Iraq invasion (2003-2004). The time resolution for our analysis is by month. For each entity and event, we extract all the articles published in NYT that mention the entity at least once. We use the dynamic topic model to derive 10 topics for each of the entities in the mentioned periods. We focus on assessing the models along the moral polarity dimension that has relatively clear-cut ground-truth for the historical incidents.

Table 2 summarizes the result per entity and event. First, all the topics identified by the topicbased source model align with the (advent of) his- torical events. For instance, the negative change in the moral polarity toward George H. W. Bush detected between 1990-07 and 1991-02 is associated with the topic of Iraq and Saddam Hussein. Comparisons on $\Delta J$ between the topic-based model and influence function indicate that these methods are equally effective in terms of the influence of the source documents ( $p=0.348$ via paired t-test), while the topic-based model significantly outperforms the random baseline $(p<0.01)$. Moreover, the topic-based model significantly outperforms the influence function and the random baseline $(p<0.05)$ in the expected coherence of the retrieved source documents (i.e., $E[H]$ ). This set of results shows that the topic-based model is on par with the established influence function retrieving influential source documents that underlie moral sentiment change, and it is significantly more ef- 
fective in selecting coherent articles relevant to the source of moral change. It is also important to note that although the influence function is designed to retrieve the most influential documents, it is computationally prohibitive to exhaustively search all possible documents, and here we applied a random search. In contrast, the topic-based model can also retrieve a set of influential documents, but it does not require an exhaustive iteration through all possible sets. Figure 3 illustrates and interprets the source analysis for the Clinton-Lewinsky Scandal.

\subsection{Application to textual source analysis of moral change in COVID-19 news}

In the final case study, we apply our framework to textual source analyses of moral change in COVID19 news. Differing from the NYT case where we focused on evaluating moral changes against known historical events, here we focus on a real-time exploratory analysis of the COVID-19 news for four entities: Donald Trump, Anthony Fauci, Andrew Cuomo, and China. ${ }^{7}$

Figure 4 shows the moral source analysis of Donald Trump. The topics selected for each change point align well with the notable incidences in COVID-19 pandemic (as annotated), suggesting how such source events can be traced in short time windows from text. The top row shows the time course of moral relevance for Donald Trump. Some relevant topic words are china, blame, disinformation, and asian. The retrieved relevant words in the middle row, reflecting a moral polarity change are flynn, ratcliffe, fbi, and investigation. The bottom row shows similar analyses for subversion which is one of the 10 moral foundations. The changing point occurs at week of 2020-05-18, and the relevant terms include george floyd, police, protest, and riot. For a more in-depth analysis, we apply the topic-based source model to all four entities. The table in Appendix C summarizes the results. Certain events during the pandemic had significant impact on how the entities are morally portrayed in the news. For example, George Floyd incident is attributed to be the source of the increase in subversion for Donald Trump. The shift happens after the week starting on May $18^{\text {th }}$, which is close to the incident date May $25^{\text {th }}$. Our model also identifies context when an entity becomes morally relevant. For example, it finds an increase in moral relevance

\footnotetext{
${ }^{7}$ We use a sliding window for change point analysis on a weekly scale, and each time point is a week starting from January, 2020.
}

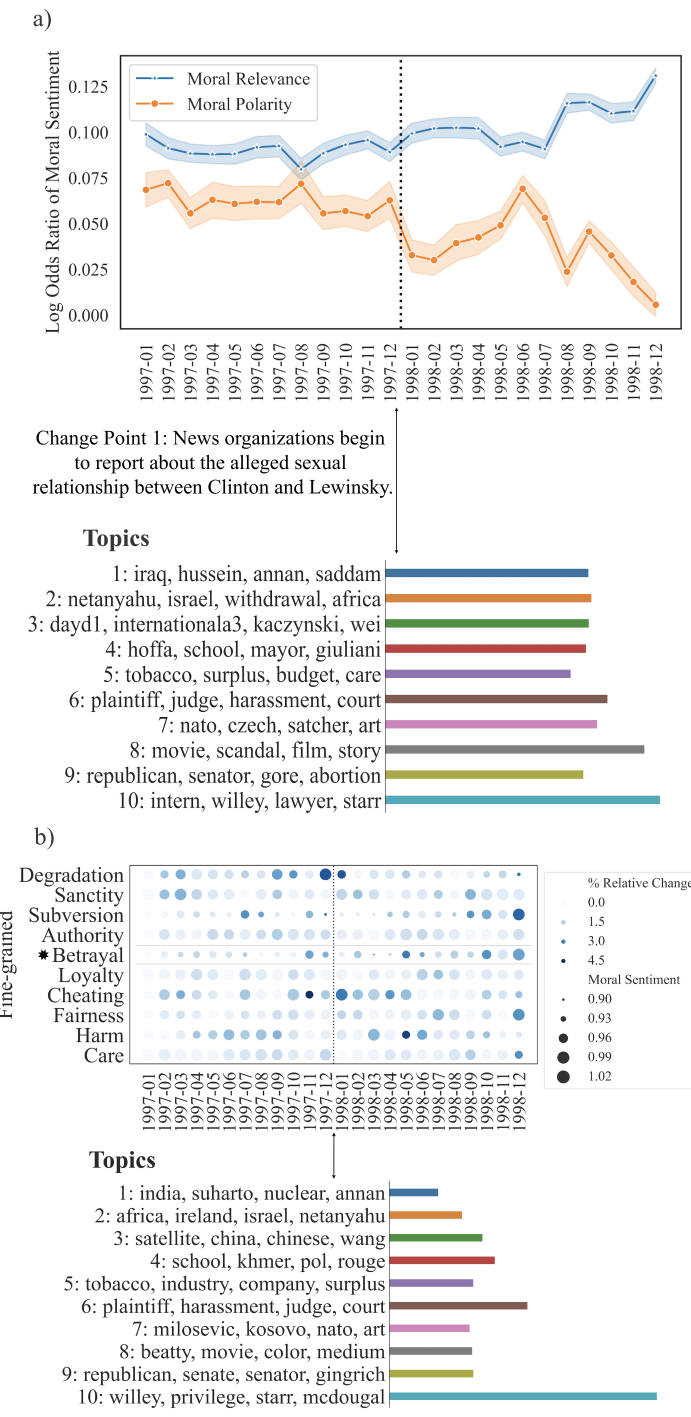

Figure 3: Textual source analysis of moral change toward Bill Clinton from 1997-1998. a) Changes in moral relevance and polarity. The vertical dashed line shows change point aligned with the start of the scandal. The bar plot below shows the topics and their relative contributions to the change. b) Fine-grained moral sentiment change toward Bill Clinton. The bottom plot shows the topical contributions in change along the Betrayal dimension from 1997-12 to 1998-04. Topic 10 is the most salient source.

for Anthony Fauci during June $22^{\text {nd }}$ to July $27^{\text {th }}$ with source topics concerning the conspiracy theories of COVID-19 treatment in social media, and the conflicts between Fauci and Trump.

\section{Discussion and conclusion}

We have presented an unsupervised framework that uses topical information to infer textual sources of moral change for entities. Our work extends existing NLP methods for moral change inference by 

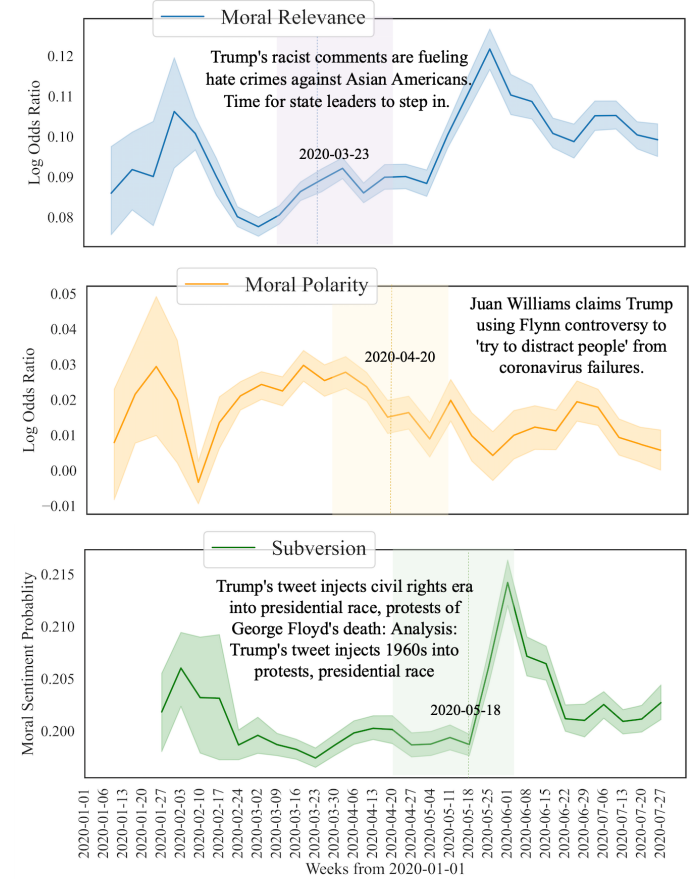

Figure 4: Textual sources of moral change toward Donald Trump in COVID-19 news. Shaded boxes show the sliding windows where the change points are detected. The topic-based model finds the most salient topic, annotated with the headline of a high-probability article.

identifying the origins of moral change over time. With evaluations on a diverse set of data, we show that our method captures both the fine-grained human moral judgments and coherent source text of moral change relevant to social events.

Our approach differs from work on general sentiment inference, partly because moral sentiment has a more fine-grained and hierarchical structure that involves inference at three different tiers, e.g., moral relevance, moral polarity (vice vs. virtue), and moral foundations. Previous work has evaluated rigorously models that capture this 3-tier moral hierarchy (Xie et al., 2019). Our framework builds on this study by characterizing the textual source of moral change at each of the three tiers. Although moral polarity can overlap with general sentiments such as good and bad, our framework captures moral sentiment beyond this dichotomous dimension. For instance, an increase in the moral relevance of an entity can be driven by an increase in moral authority, which may or may not involve any positive or negative sentiment (see Appendix $\mathrm{C}$ for examples). In this respect, moral sentiment captured by our framework can be dissociated with sentiment portrayed in the traditional NLP literature.
Our work makes minimal claims about the causes of moral change. Our focus here is to identify salient topics as the source of moral sentiment change. This topical information can be a proxy to world events that trigger changes in moral perception toward an entity. Identifying the causes of moral change beyond textual sources studied here can be an exciting yet challenging direction.

Our framework also offers opportunities for further exploration of entity-based moral sentiment change. Future work may explore how different media platforms vary in the moral sentiments that they convey towards entities (e.g., public figures) and the sources of this variation.

\section{Acknowledgements}

We thank Graeme Hirst, Jennifer Stellar, Lea Frermann, and Matthew Fienberg for their feedback on our manuscript. AR is funded partly by a Schwartz Reisman Institute for Technology and Society Graduate Fellowship. This work was supported by a NSERC Discovery Grant RGPIN-201805872, a SSHRC Insight Grant \#435190272, and an Ontario ERA Award to YX.

\section{Broader impact and ethical statement}

Our study applies natural language processing to the source identification of moral change triggered by high-impact historical and contemporary social events. The framework we have developed provides an automated and scalable tool for interdisciplinary scholars including computational linguists, psychologists, and social scientists to quantitatively investigate the origins of moral change.

Some of our analyses on social media and news articles can be considered sensitive. The results that we report here do not represent our personal beliefs or opinions and could potentially be influenced by the biases contained in the datasets.

\section{References}

Aristotle, W. D. Ross, and Lesley Brown. 2009. The Nicomachean ethics. Oxford University Press.

David M Blei and John D Lafferty. 2006. Dynamic topic models. In Proceedings of the 23rd International Conference on Machine Learning, pages 113120.

Paul Bloom. 2010. How do morals change? Nature, 464(7288):490. 
Marc-Etienne Brunet, Colleen Alkalay-Houlihan, Ashton Anderson, and Richard Zemel. 2019. Understanding the origins of bias in word embeddings. In Proceedings of the 36th International Conference on Machine Learning, volume 97 of Proceedings of Machine Learning Research, pages 803-811. PMLR.

R Dennis Cook and Sanford Weisberg. 1980. Characterizations of an empirical influence function for detecting influential cases in regression. Technometrics, 22(4):495-508.

Jacob Devlin, Ming-Wei Chang, Kenton Lee, and Kristina Toutanova. 2019. BERT: Pre-training of deep bidirectional transformers for language understanding. In Proceedings of the 2019 Conference of the North American Chapter of the Association for Computational Linguistics: Human Language Technologies, Volume 1 (Long and Short Papers), pages 4171-4186, Minneapolis, Minnesota. Association for Computational Linguistics.

Maxwell Forbes, Jena D Hwang, Vered Shwartz, Maarten Sap, and Yejin Choi. 2020. Social chemistry 101: Learning to reason about social and moral norms. In Proceedings of the 2020 Conference on Empirical Methods in Natural Language Processing.

Justin Garten, Reihane Boghrati, Joe Hoover, Kate M Johnson, and Morteza Dehghani. 2016. Morality between the lines: Detecting moral sentiment in text. In Proceedings of IJCAI 2016 workshop on Computational Modeling of Attitudes.

Jesse Graham, Jonathan Haidt, Sena Koleva, Matt Motyl, Ravi Iyer, Sean P Wojcik, and Peter H Ditto. 2013. Moral foundations theory: The pragmatic validity of moral pluralism. In Advances in Experimental Social Psychology, volume 47, pages 55-130. Elsevier.

Jesse Graham, Jonathan Haidt, and Brian A Nosek. 2009. Liberals and conservatives rely on different sets of moral foundations. Journal of Personality and Social Psychology, 96(5):1029.

Jonathan Haidt. 2001. The emotional dog and its rational tail: a social intuitionist approach to moral judgment. Psychological Review, 108(4):814.

Joe Hoover, Gwenyth Portillo-Wightman, Leigh Yeh, Shreya Havaldar, Aida Mostafazadeh Davani, Ying Lin, Brendan Kennedy, Mohammad Atari, Zahra Kamel, Madelyn Mendlen, et al. 2020. Moral Foundations Twitter Corpus: A collection of 35k tweets annotated for moral sentiment. Social Psychological and Personality Science, 11(8):1057-1071.

David Hume. 1739. A treatise of human nature. Clarendon Press.

Sophie Jentzsch, Patrick Schramowski, Constantin Rothkopf, and Kristian Kersting. 2019. The Moral Choice Machine: Semantics derived automatically from language corpora contain human-like moral choices. In Proceedings of the 2nd AAAI/ACM Conference on AI, Ethics, and Society. Palo Alto (California): Association for the Advancement of Artificial Intelligence.

Immanuel Kant. 1785. Groundwork for the Metaphysics of Morals. Oxford University Press.

Pang Wei Koh and Percy Liang. 2017. Understanding black-box predictions via influence functions. In Proceedings of the 34th International Conference on Machine Learning - Volume 70, ICML'17, page 1885-1894. JMLR.org.

Lawrence Kohlberg. 1969. Stage and Sequence; The Cognitive-developmental Approach to Socialization. Rand McNally.

Lawrence Kohlberg and Richard H Hersh. 1977. Moral development: A review of the theory. Theory into Practice, 16(2):53-59.

Vivek Kulkarni, Rami Al-Rfou, Bryan Perozzi, and Steven Skiena. 2015. Statistically significant detection of linguistic change. Proceedings of the 24th International Conference on World Wide Web, pages 625-635.

Ying Lin, Joe Hoover, Gwenyth Portillo-Wightman, Christina Park, Morteza Dehghani, and Heng Ji. 2018. Acquiring background knowledge to improve moral value prediction. In 2018 IEEE/ACM International Conference on Advances in Social Networks Analysis and Mining (ASONAM), pages 552-559. IEEE.

Nicholas Lourie, Ronan Le Bras, and Yejin Choi. 2020. Scruples: A corpus of community ethical judgments on 32,000 real-life anecdotes. arXiv preprint arXiv:2008.09094.

Tomas Mikolov, Kai Chen, Greg S. Corrado, and Jeffrey Dean. 2013. Efficient estimation of word representations in vector space. In International Conference on Learning Representations, 2013a.

Marlon Mooijman, Joe Hoover, Ying Lin, Heng Ji, and Morteza Dehghani. 2018. Moralization in social networks and the emergence of violence during protests. Nature Human Behaviour, 2(6):389-396.

Friedrich Wilhelm Nietzsche. 1887. Zur genealogie der moral: Eine streitschrift. CG Naumann.

Jean Piaget. 1932. The moral judgment of the child. London: Routledge and Kegan Paul.

David A Pizarro and Paul Bloom. 2003. The intelligence of the moral intuitions: A comment on Haidt (2001). Psychological review, 110(1):193-6; discussion 197-8.

Plato and Allan Bloom. 1968. The Republic. New York. 
Evan Sandhaus. 2008. The New York Times annotated corpus. Linguistic Data Consortium, Philadelphia, 6(12):e26752.

Patrick Schramowski, Cigdem Turan, Sophie Jentzsch, Constantin Rothkopf, and Kristian Kersting. 2019. Bert has a moral compass: Improvements of ethical and moral values of machines. arXiv preprint arXiv:1912.05238.

Adam Smith. 1759. The Theory of Moral Sentiments. McMaster University Archive for the History of Economic Thought.

Jing Yi Xie, Renato Ferreira Pinto Junior, Graeme Hirst, and Yang Xu. 2019. Text-based inference of moral sentiment change. In Proceedings of the 2019 Conference on Empirical Methods in Natural Language Processing and the 9th International Joint Conference on Natural Language Processing (EMNLP-IJCNLP), pages 4654-4663, Hong Kong, China. Association for Computational Linguistics.

Jing Yi Xie, Graeme Hirst, and Yang Xu. 2020. Contextualized moral inference. arXiv preprint arXiv:2008.10762. 


\section{A Details of data pre-processing}

We take the following steps in pre-processing for a document and a query entity: 1) We find all the mentions of the entity in the document using the coreference resolution module specified in the paper. 2) We discard all the sentences in the document that do not include any mention of the entity. 3) We lemmatize the tokens in the documents using the spaCy English model. 4) We remove the entity with all its mentions, function words, and all the other words if they are classified as morally irrelevant using the centroid model following Xie et al. (2019). 5) We take an average of the word embeddings of the remaining tokens to derive the feature vector of the document. If the task is based on the BERT embeddings, we skip step 4 , but to derive the vector representation of the document, we take an average of the tokens that pass through step 4 in the final layer.

In Xie et al. (2019), the centroid model compares the Euclidean distance of an embedded input to the center of morally relevant words and the center of morally neutral words. The distances are then transformed to probabilities using a softmax function.

Our framework models moral sentiment as a hierarchical concept under the three tiers of 1) moral relevance, 2) moral polarity, and 3) 10 finegrained moral foundation categories. All the calculations for the moral sentiment dimensions are performed on the documents that satisfy this hierarchical framework. For instance, when estimating the moral polarity, the documents classified as morally irrelevant are discarded. Similarly, for each fine-grained category, we discard documents with an opposing moral polarity. 


\section{B Comparison of source news articles retrieved for Bill Clinton case study}

Headlines of news articles retrieved by topic-based source model

Lawyers for Jones Get More Response Time

Whispered Secrets Start a Loud Debate

Starr Is Right to Question White House Aide; Having It Both Ways

Lewinsky's Bookstore Purchases Are Now Subject of a Subpoena

Headlines of news articles retrieved by influence function method

A Waggish Tale In Washington...

Starr Subpoenas Notes and Case Files of Lewinsky's Former Lawyer

Would Punishing Iraq Carry Too High a Price? Vietnam's Lesson

Day of Facing the Nation, Meeting the Press, Etc.

Headlines of news articles retrieved by random baseline

Public Radio Hosts Drop In and Maybe Stay Too Long

Book Agent Advised Taping Accusations

After Derailing Trade Bill, Labor Sets Ambitious Goals

Yes, a Surplus Would Help, But Tough Choices Remain

Table 3: Headlines of 4 randomly sampled news articles retrieved by the three models as source for moral sentiment change toward Bill Clinton during ClintonLewinsky Scandal (1997-12 to 1998-03). 


\section{Additional results from moral change source analysis for entities in COVID-19 news}

\begin{tabular}{|c|c|c|c|c|}
\hline Entity & Initial point & $\begin{array}{l}\text { Ending } \\
\text { point }\end{array}$ & $\begin{array}{l}\text { Moral } \\
\text { Dimension }\end{array}$ & Topic Words \\
\hline \multirow[t]{4}{*}{ Trump } & $2020-03-23$ & 2020-04-20 & Relevance $\uparrow$ & $\begin{array}{l}\text { conspiracy, xenophobic, disinformation } \\
\text { china, originate, blame, asian }\end{array}$ \\
\hline & $2020-05-18$ & 2020-06-22 & Relevance $\uparrow$ & $\begin{array}{l}\text { juneteenth, police, black, floyd, racism, } \\
\text { brutality, protest, racial, minneapolis }\end{array}$ \\
\hline & 2020-04-20 & 2020-05-11 & Polarity $\downarrow$ & $\begin{array}{l}\text { flynn, muir, mcenany, miller, obama } \\
\text { collusion, ratcliffe, whistleblower }\end{array}$ \\
\hline & $2020-05-18$ & 2020-06-01 & Subversion $\uparrow$ & $\begin{array}{l}\text { killing, george floyd, protest, black } \\
\text { minneapolis, peaceful, racism, murder }\end{array}$ \\
\hline \multirow[t]{2}{*}{ Fauci } & 2020-06-22 & $2020-07-27$ & Relevance $\uparrow$ & $\begin{array}{l}\text { sinclair, twitter, mikovit, conspiracy } \\
\text { facebook, vaccine, mask, video }\end{array}$ \\
\hline & 2020-06-29 & 2020-07-20 & Fairness $\uparrow$ & $\begin{array}{l}\text { disapprove, statue, cain, goya, gop } \\
\text { electoral, biden, campaign, tulsa }\end{array}$ \\
\hline \multirow[t]{4}{*}{ Cuomo } & $2020-05-11$ & 2020-06-01 & Relevance $\uparrow$ & $\begin{array}{l}\text { george floyd, cop, demonstration } \\
\text { injustice, black, peaceful, protest, racism }\end{array}$ \\
\hline & 2020-03-30 & 2020-05-04 & Polarity $\downarrow$ & $\begin{array}{l}\text { 14-day, death, flatten, epicenter } \\
\text { lockdown, peak, social distancing, reopen }\end{array}$ \\
\hline & $2020-05-25$ & $2020-06-22$ & Polarity $\downarrow$ & $\begin{array}{l}\text { george floyd, cop, demonstration } \\
\text { injustice, black, peaceful, protest, racism }\end{array}$ \\
\hline & $2020-03-23$ & 2020-04-13 & Cheating $\downarrow$ & $\begin{array}{l}\text { 14-day, death, flatten, epicenter } \\
\text { lockdown, peak, social distancing, reopen }\end{array}$ \\
\hline \multirow[t]{5}{*}{ China } & $2020-03-23$ & 2020-04-20 & Relevance $\uparrow$ & $\begin{array}{l}\text { blame, disinformation, trump, conspiracy } \\
\text { accountable, downplay }\end{array}$ \\
\hline & 2020-05-11 & $2020-05-25$ & Relevance $\uparrow$ & $\begin{array}{l}\text { hong kong, freedom, democracy, } \\
\text { economic, tension, territory }\end{array}$ \\
\hline & $2020-02-24$ & $2020-03-23$ & Polarity $\uparrow$ & $\begin{array}{l}\text { iran, ban, passenger, flight } \\
\text { quarantine, cruise, case, korea, japan }\end{array}$ \\
\hline & $2020-05-11$ & $2020-05-25$ & Polarity $\uparrow$ & $\begin{array}{l}\text { hong kong, freedom, democracy } \\
\text { economic, tension, territory }\end{array}$ \\
\hline & 2020-02-24 & 2020-03-23 & Authority $\uparrow$ & $\begin{array}{l}\text { flu, disease, sick, test, care, influenza } \\
\text { cough, respiratory, ventilator, covid-19 }\end{array}$ \\
\hline
\end{tabular}

Table 4: Source analyses of moral sentiment change of entities in COVID-19 along different moral dimensions. Arrows indicate the polarities of change. The most salient words from the source topics are shown for each entity. 